Erratum

\title{
Erratum: Xie, Y.Y.; et al. GRACE-Based Terrestrial Water Storage in Northwest China: Changes and Causes. Remote Sens. 2018, 10, 1163
}

\author{
Yangyang Xie ${ }^{1,2}$, Shengzhi Huang ${ }^{1, *}$, Saiyan Liu ${ }^{1}$, Guoyong Leng ${ }^{3}$, Jian Peng ${ }^{4,5,6}$ (1), \\ Qiang Huang ${ }^{1}$ and Pei $\mathrm{Li}^{1}$ \\ 1 State Key Laboratory Base of Eco-Hydraulic Engineering in Arid Area, Xi'an University of Technology, \\ Xi'an 710048, China; xieyang_yang_cool@126.com (Y.X.); ziyuanxxb@126.com (S.L.); \\ sy-sj@xaut.edu.cn (Q.H.); m18792577057@163.com (P.L.) \\ 2 School of Hydraulic Energy and Power Engineering, Yangzhou University, Yangzhou 225000, China \\ 3 Environmental Change Institute, University of Oxford, Oxford OX1 3QY, UK; guoyong.leng@ouce.ox.ac.uk \\ 4 School of Geography and the Environment, University of Oxford, Oxford OX1 3QY, UK; \\ jian.peng@ouce.ox.ac.uk \\ 5 Department of Geography, University of Munich (LMU), 80333 Munich, Germany \\ 6 Max Planck Institute for Meteorology, 20146 Hamburg, Germany \\ * Correspondence: huangshengzhi7788@126.com; Tel.: +86-156-8606-0577
}

Received: 31 July 2018; Accepted: 3 August 2018; Published: 15 August 2018

The authors wish to make a correction to their paper [1]. After the final proofreading, they realized that the affiliation was incorrect due to their carelessness. Yangyang Xie, the first author of this paper, was a PhD student at Xi'an University of Technology. After his graduation, he worked at Yangzhou University. However, the work for this accepted paper was mostly finished at Xi'an University of Technology. Therefore, they want to change the original affiliations, and all of the authors have confirmed and agreed with the application of the changes. The following affiliations should be replaced with the new ones shown below them.

Yangyang Xie ${ }^{1}$, Shengzhi Huang ${ }^{2, *}$, Saiyan Liu ${ }^{2}$, Guoyong Leng ${ }^{3}$, Jian Peng ${ }^{4,5,6}$, Qiang Huang ${ }^{2}$ and Pei $\mathrm{Li}^{2}$

1 School of Hydraulic Energy and Power Engineering, Yangzhou University, Yangzhou 225000, China; xieyang_yang_cool@126.com

2 State Key Laboratory Base of Eco-Hydraulic Engineering in Arid Area, Xi'an University of Technology, Xi'an 710048, China; ziyuanxxb@126.com (S.L.); sy-sj@xaut.edu.cn (Q.H.); m18792577057@163.com (P.L.)

3 Environmental Change Institute, University of Oxford, Oxford OX1 3QY, UK; guoyong.leng@ouce.ox.ac.uk

4 School of Geography and the Environment, University of Oxford, Oxford OX1 3QY, UK; jian.peng@ouce.ox.ac.uk

5 Department of Geography, University of Munich (LMU), 80333 Munich, Germany

6 Max Planck Institute for Meteorology, 20146 Hamburg, Germany

* Correspondence: huangshengzhi7788@126.com; Tel.: +86-156-8606-0577 


\section{Yangyang Xie ${ }^{1,2}$, Shengzhi Huang ${ }^{1, *}$, Saiyan Liu ${ }^{1}$, Guoyong Leng ${ }^{3}$, Jian Peng ${ }^{4,5,6}$, Qiang Huang ${ }^{1}$} and Pei $\mathrm{Li}^{1}$

1 State Key Laboratory Base of Eco-Hydraulic Engineering in Arid Area, Xi'an University of Technology, Xi'an 710048, China; xieyang_yang_cool@126.com (Y.X.); ziyuanxxb@126.com (S.L.); sy-sj@xaut.edu.cn (Q.H.); m18792577057@163.com (P.L.)

2 School of Hydraulic Energy and Power Engineering, Yangzhou University, Yangzhou 225000, China

3 Environmental Change Institute, University of Oxford, Oxford OX1 3QY, UK; guoyong.leng@ouce.ox.ac.uk

4 School of Geography and the Environment, University of Oxford, Oxford OX1 3QY, UK; jian.peng@ouce.ox.ac.uk

5 Department of Geography, University of Munich (LMU), 80333 Munich, Germany

6 Max Planck Institute for Meteorology, 20146 Hamburg, Germany

* $\quad$ Correspondence: huangshengzhi7788@126.com; Tel.: +86-156-8606-0577

The authors would like to apologize for any inconvenience caused to the readers by these changes. The changes do not affect the scientific results. The manuscript will be updated and the original will remain online on the article webpage, with a reference to this correction.

\section{References}

1. Xie, Y.; Huang, S.; Liu, S.; Leng, G.; Peng, J.; Huang, Q.; Li, P. GRACE-Based Terrestrial Water Storage in Northwest China: Changes and Causes. Remote Sens. 2018, 10, 1163. [CrossRef] 